\title{
Nanofabrication with Ultrasonic Nanoimprint Lithography
}

\author{
Chien-Hung Lin \\ Institute of Microelectromechanical System \\ National Tsing Hua University \\ Hsinchu, Taiwan \\ d929208@oz.nthu.edu.tw
}

\author{
Rongshun Chen \\ Institute of Microelectromechanical System \\ National Tsing Hua University \\ Hsinchu, Taiwan \\ rchen $a$ pme.nthu.edu.tw
}

\begin{abstract}
The ultrasonic nanoimprint lithography method provides high productivity, energy efficiency, and low cost. We propose a new nanoimprint lithography technique based on direct heating of the polymer resist by ultrasonic vibrations instead of using heaters in conventional nanoimprint lithography. In this paper we demonstrate that ultrasonic stack design is very important to ultrasonic nanoimprint lithography system. Since properties of ultrasonic vibrations obviously affect imprint results. If the diameter of the horn is greater than twice times of the size of the mold, the transferred patterns are completely replicated from the mold. To the best imprint results, the imprinted force, ultrasonic power, and ultrasonic time should be adjusted.
\end{abstract}

Keywords-component: nanofabrication; ultrasonic; nanoimprint.

\section{INTRODUCTION}

Nanoimprint lithography has been developed for ten years. It is a novel method of fabricating nanometer scale patterns. There are a lot of researchers to develop many different variations and implementations [1]. Recently, nanoimprint lithography has been included in the International Technology Roadmap for Semiconductors (ITRS 2003 Edition) for the 32 nm node [2].

Thermal nanoimprint lithography, the earliest and most mature nanoimprint lithography, requires thermal cycles between $140^{\circ} \mathrm{C}$ and $180^{\circ} \mathrm{C}$ to heat up above the glass transition temperature of the imprinted resist polymer and high pressure during the hot embossing procedure. The pattern on the mold is pressed into the melt polymer film on the substrate. After being cooled down, the mold is separated from the substrate and the pattern resist is left on the substrate. However, it spends a lot of time to heat and cool. This work presents a new approach of nanofabrication with the ultrasonic nanoimprint lithography [3]. It bases on direct heating of the resist polymer by ultrasonic vibrations instead of using heaters in thermal nanoimprint lithography. An ultrasonic source is located on the top of mold to generate high frequency vibrations causing the increase of temperature to soften and to melt the thermoplastic polymer [4]. The ultrasonic waves can rapidly increase the temperature of polymer to significantly reduce the process time. Our results may provide the potential to become a novel nanoimprint method with high productivity, energy efficiency, and low cost.

\section{ULTRASONIC NANOIMPRINT LITHOGRAPHY}

A. Ultrasonic vibrations

A typical ultrasonic stack consists of a transducer (or converter), booster, and horn. Ultrasonic vibrations are generated by bolt-clamped Langevin-type (sandwich) transducers, widely used as the vibratory source of ultrasonic power due to its high efficiency. The piezoelectric ceramic rings are sandwiched by two metal end-blocks and clamped with a screw bolt. A booster can adjust vibratory output from transducer and transfer energy to the horn. Furthermore, the booster provides flange for mounting the ultrasonic stack to support structure. A horn can contact the load and deliver power to the load. Each of components is designed to vibrate in a longitudinal mode at the specified frequency. Since the output surface of the horn should contact with the mold and the substrate, uniform output amplitude of horn is necessary. The dimensions of each component have been conducted with finite element method to calculate its resonant frequency and vibration mode, and predict its dynamic performance.

In our design, the resonant frequency is $30 \mathrm{kHz}$. The transducer includes a pair of piezoelectric rings, PZT-4, between two end-blocks. The material of the transducer, booster, and horn is Aluminum alloy 7075, whose properties are listed in Table 1. After simulated results, ultrasonic stack is show in Fig. 1 and its resonant frequency is $30.02 \mathrm{~Hz}$.

TABLE I. MATERIAL PROPERTIES OF ALUMNUM ALLOY 7075

\begin{tabular}{|c|c|c|}
\hline \multicolumn{3}{|c|}{ Aluminum alloy 7075 } \\
\hline Young's modulus & Density & Poisson's Ratio \\
\hline $71.7 \mathrm{GPa}$ & $2810 \mathrm{~kg} / \mathrm{m}^{3}$ & 0.33 \\
\hline
\end{tabular}

\section{B. System}

The apparatus of ultrasonic nanoimprint lithography consists of two components as shown in Fig. 2. The one is the ultrasonic system that is the core of ultrasonic nanoimprint lithography. It includes a power supply and ultrasonic stack. Since the resonant frequency of ultrasonic vibrations is designed to $30 \mathrm{kHz}$ in the longitudinal mode, the electrical signal, which closely matches the resonant frequency of the stack, must be applied from the power supply to transducer. 
The other is the imprint machine. The pneumatic cylinder is controlled by a series of a manual valve used as a switch for pressing or separating process. The regulator determines imprinted force by using output flow control valve and the load cell (LPX 250, PT Ltd.) can be used to measure the imprinted force that is registered in $\mathrm{kg}$ on the digital display.

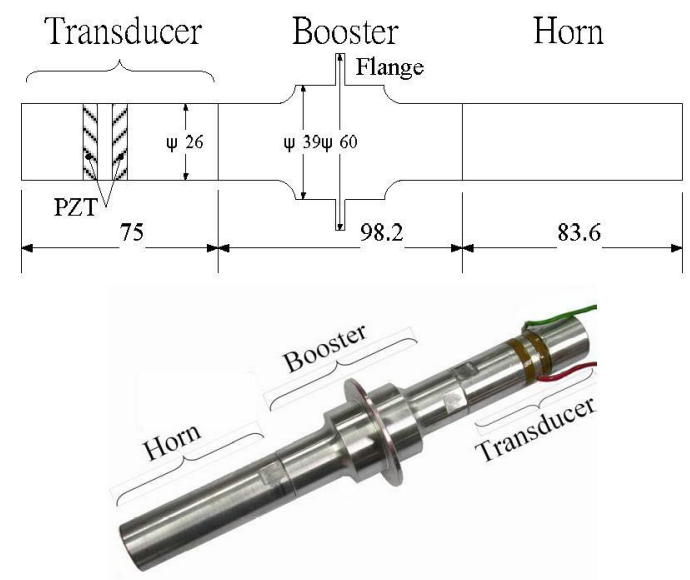

Figure 1. Ultrasonic stack consists of a transducer, booster, and horn.

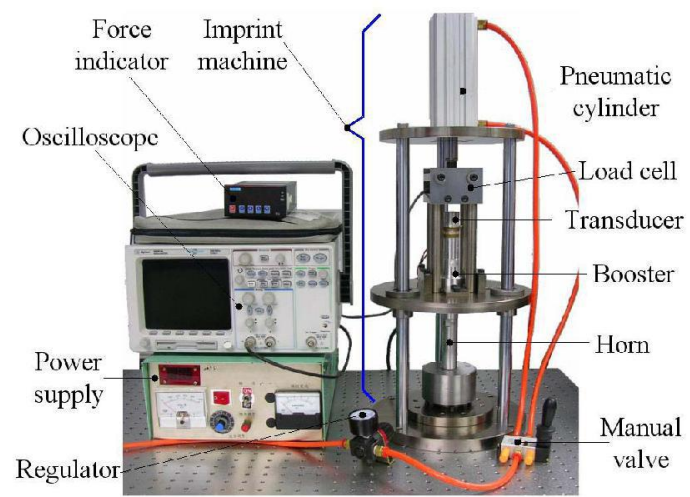

Figure 2. Apparatus of ultrasonic nanoimprint lithography.

\section{Process}

The schematic process of ultrasonic nanoimprint lithography is showed in Fig. 3. The first, the thin thermoplastic polymer film is spin-coated on a substrate and has a thickness similar to the required structure height. The second, the mold and the substrate are put on the substrate holding of the machine press after alignment. The pneumatic cylinder provides the horn to press mold with very slow speed, in which the mold is uniformly contacted with the polymer film, placed on the top of substrate. The third, the sinusoidal electrical signal is applied to the transducer to provide vibrations for a few second. Through the intermolecular friction, the generated heat, which raises temperature higher than the glass transition temperature, is required to reduce locally viscosity of polymer. During this period, the force is kept constant to allow the polymer to flow into the cavities of mold. The fourth, the ultrasonics stops operating, while the applied force still keeps constant until the mold cooling down. The fifth, the applied force is released and the mold is separated from the polymer film. The final, the residual polymer film is removed by using anisotropic etching process to produce the nanopatterns on the substrate. Fig. 3 displays the histories of imprinted force and ultrasonics in the process.

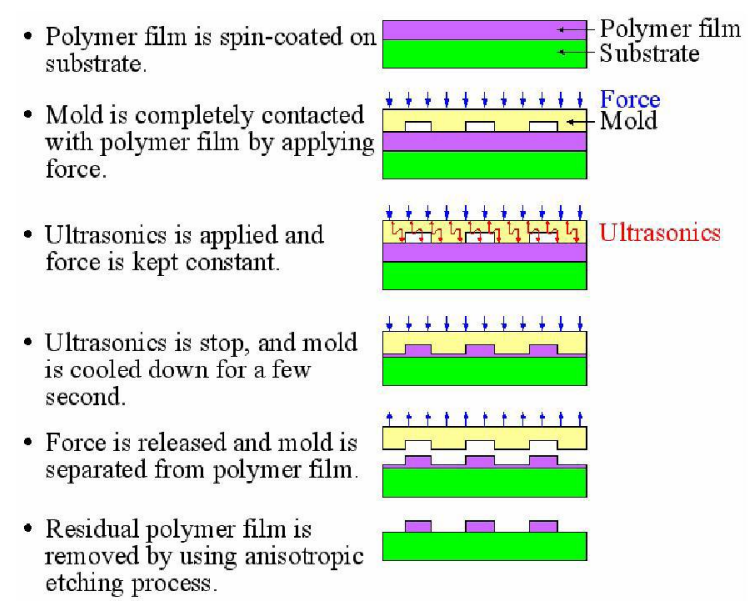

Figure 3. Schematic process of ultrasonic nanoimprint lithogaphy.

III. RESULTS

\section{A. Mold Fabrication}

The mold was fabricated by I-line photolithography and ICP-RIE on a silicon substrate. The patterns of the mold consist of an array of pillars whose widths range from $500 \mathrm{~nm}$ to $5 \mu \mathrm{m}$ and depths are $300 \mathrm{~nm}$. Fig. 4 shows the AFM image and SEM micrograph of the array of pillars on the mold. Then, the substrate was treated with trichloro (1,1,2,2-perfluoroctyl) silane as a anti-adhesive laver by vapor deposition method. Finally, the substrate is cut into small portions whose area is 10 $\mathrm{mm}$ by $10 \mathrm{~mm}$ to obtain molds.

\section{B. Imprinted Polymer}

Imprinted polymer is a commercially available thermoplastic polymer mr-I-8030 (Micro Resist Technology Gmbh, $\mathrm{Tg}=115^{\circ} \mathrm{C}$ ) in our experiments. The silicon wafer would follow RCA clean procedure, spin-coat with the polymer and bake on a hotplate at $140{ }^{\circ} \mathrm{C}$ for $3 \mathrm{~min}$. The thickness of polymer film is about $300 \mathrm{~nm}$.

\section{Imprint Results}

For ultrasonic nanoimprint lithography, the imprint force, ultrasonic power, and ultrasonic time are very important. When the imprinted force of $70 \mathrm{kgf}$, ultrasonic power of $240 \mathrm{~W}$, and the ultrasonic time of 15 seconds are applied in process, the optical micrographs of imprinted result are shown in Fig. 5. The transferred patterns are completely replicated from the mold. But there is some defect in the cavities border. In Fig. 6 of AFM image, convex profile is clearly appeared in the cavities border. Because the localized heat affective zones are concentrated on the salient of the mold during ultrasonic embossing, the flow of the squeezed polymer is too fast. That means the ultrasonic energy makes the temperature of polymer rise in region of the convex feature on the mold. The polymer 
flow in the mold cavities cannot be mixed very well in high temperature gradient. It seems that the ultrasonic power and imprint force should be enhanced to provide uniform temperature field and to make polymer fill in the mold cavities.
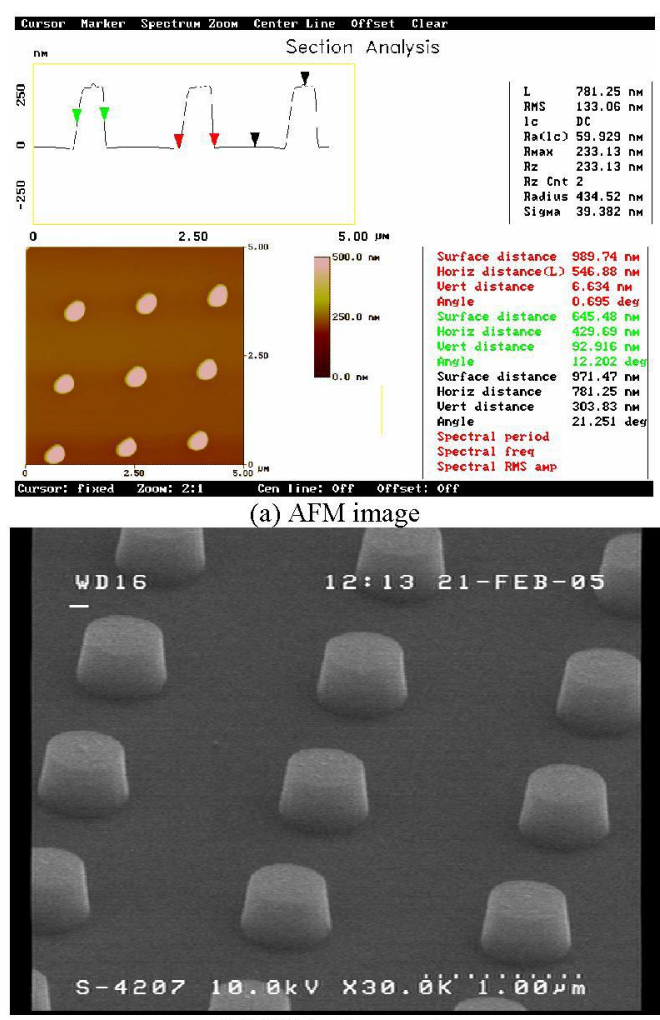

(b) SEM micrograph

Figure 4. (a) AFM imageas and (b) SEM micrograph of mold consisting array of pillars.

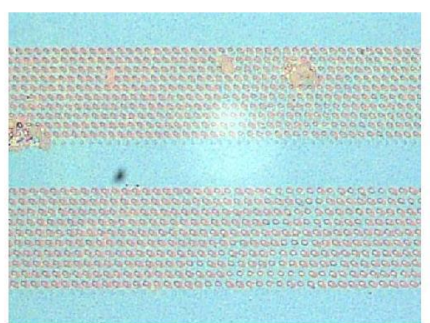

(a) $0.9 \mu \mathrm{m}$ and $1 \mu \mathrm{m}$

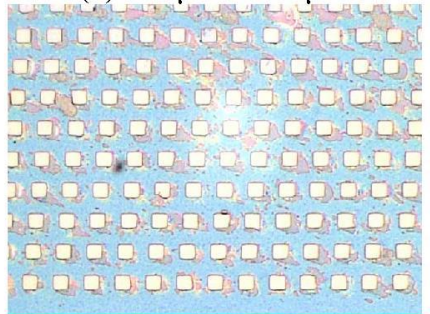

(c) $3 \mu \mathrm{m}$

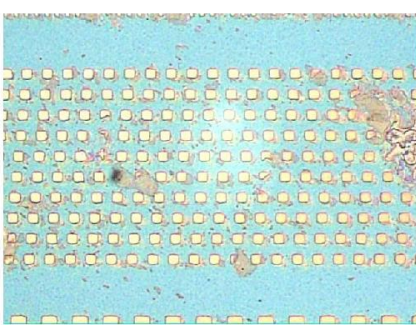

(b) $2 \mu \mathrm{m}$

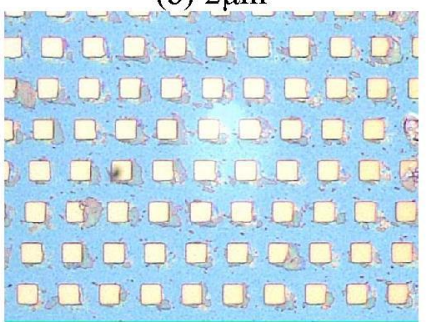

(d) $4 \mu \mathrm{m}$
Figure 5. Optical micrographs of imprinted result with sizes of (a) $0.9 \mu \mathrm{m}$ and $1 \mu \mathrm{m}$, (b) $2 \mu \mathrm{m}$ (c) $3 \mu \mathrm{m}$, and (d) $4 \mu \mathrm{m}$.

The uniformity of vibratory amplitude should affect the properties of imprinted results. In our ultrasonic stack, the diameter of the horn is $26 \mathrm{~mm}$, and the amplitude of vibrations doesn't amplify. In a longitudinal mode at resonant frequency of $30 \mathrm{kHz}$, the amplitude output of horn can predict by the finite element method and is normalized by its maximum amplitude as shown in Fig. 7. The longitudinal normalization amplitude decreases with the distance from the center of the horn. Although the longitudinal amplitude is greater than 3 order of the lateral amplitude, the lateral normalization amplitude increases with the distance from the center of the horn. The lateral amplitude would make transferred patterns not uniform and lose accuracy. In previous experiment, the diameter of the horn was $15 \mathrm{~mm}$, and it was close to the size of the mold is $10 \mathrm{~mm}$ by $10 \mathrm{~mm}$. So this study is better than the previous imprint result as shown in Fig. 8. And it is seen that some residual polymer has stuck to the mold.

The imprinted force will affect the vibratory amplitude and the flow of imprinted polymer during ultrasonic operating. Low imprinted force may result in loose contact between the mold and the polymer film causing poor transmission of ultrasonic energy. On the other hand, if the imprinted force is too high, the heat affect zones (HAZ), where are contact between the protrusion features on the mold and the polymer, have high temperature gradient. So it is a trade-off. In process, the imprinted force and ultrasonic power may be programmed to get the best imprint results.

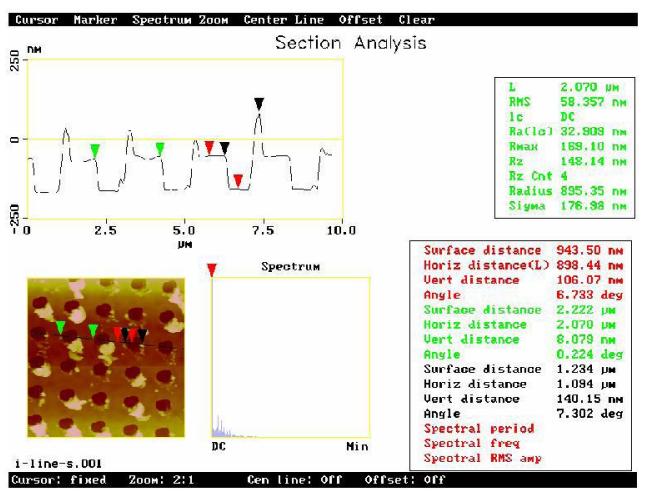

Figure 6. AFM image of imprint result.

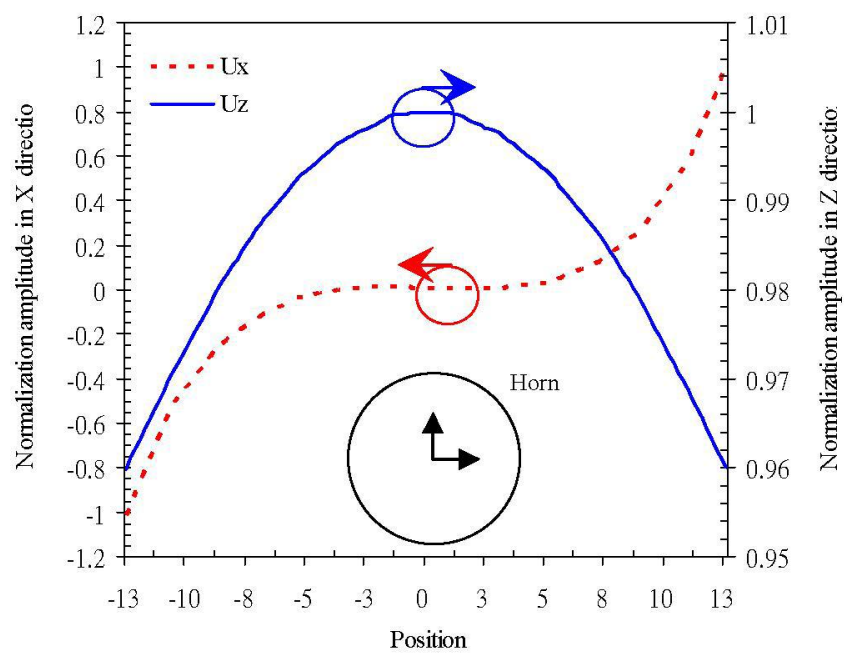

Figure 7. Amplitude output of horn by finite element method. 


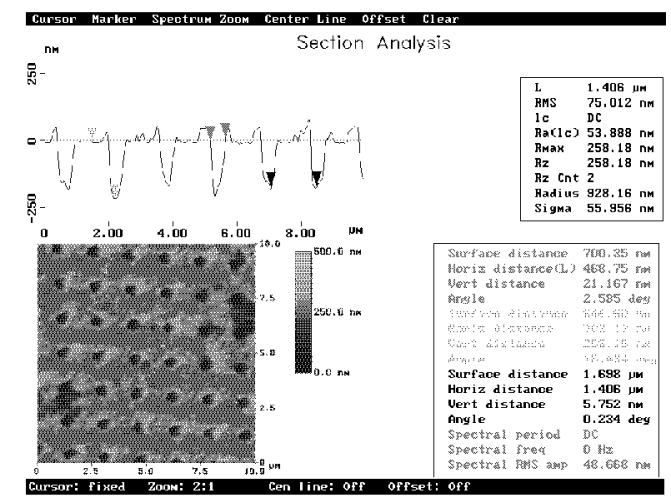

Figure 8. AFM images of imprint result in preious study with horn diameter of $15 \mathrm{~mm}$.

\section{CONCLUSION}

A imprint apparatus using ultrasonic vibrations as heaters was designed to produce the imprinted patterns on the polymer. We showed that ultrasonic stack design is very important to ultrasonic nanoimprint lithography system. The uniform ultrasonic vibrations obviously affect imprint results. If the diameter of the horn is greater than twice times of the size of the mold, the transferred patterns are completely replicated from the mold. The imprinted force, ultrasonic power, and ultrasonic time should be adjusted and programmed to get the best imprint results. As a result, the ultrasonic nanoimprint lithography has the potential to become a novel nanofabrication with high productivity, energy efficiency, and low cost.

\section{ACKNOWLEDGMENT}

We are grateful for the support of the National Science Council of Taiwan, R.O.C., by grant number NSC 94-2212-E007-005.

\section{REFERENCES}

[1] L. J. Guo, "Recent progress in nanoimprint technology and its applications," Journal of Physies D: Applied Physics, vol. 37, no. 11, pp. R123-R141, 2004

[2] http://public.itrs.net/.

[3] C. H. Lin and R. Chen, "Ultrasonic nanoimprint lithography: a new approach to nanopatterning," Journal of Microlithography, Microfabrication and Microsystems, vol. 5, no. 1, pp. 011003, 2006.

[4] M. N. Tolunay, P. R. Dawson, and K. K. Wang, "Heating and bonding mechanisms in ultrasonic welding of thermoplastics," Polymer Engineering and Science, vol. 23, no. 13, pp. 726-733, 2004. 